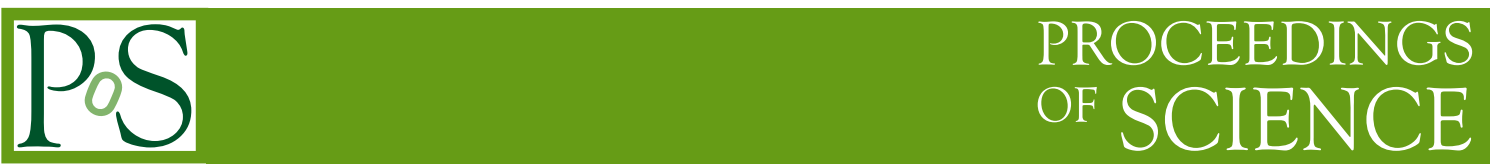

\title{
Pion form factors in two-flavor QCD
}

\section{JLQCD collaboration:}

S. Hashimoto ${ }^{a, b * \dagger}$, S. Aoki ${ }^{c}$, M. Fukugita ${ }^{d}$, K.-I. Ishikawa $^{e}$, N. Ishizuka ${ }^{f, c}$, Y. Iwasaki $^{c}$, K. Kanaya ${ }^{c}$, T. Kaneko ${ }^{a, b}$, Y. Kuramashi ${ }^{f, c}$, M. Okawa ${ }^{e}$, N. Tsutsui ${ }^{a}$, A. Ukawa ${ }^{f, c}$, N. Yamada ${ }^{a, b}$, T. Yoshiéf ${ }^{f}$

${ }^{a}$ High Energy Accelerator Research Organization (KEK), Tsukuba 305-0801, Japan;

${ }^{b}$ School of High Energy Accelerator Science, The Graduate University for Advanced Studies (Sokendai), Tsukuba 305-0801, Japan.

${ }^{c}$ Graduate School of Pure and Applied Sciences, University of Tsukuba, Tsukuba 305-8571, Japan.

${ }^{d}$ Institite for Cosmic Ray Research, University of Tokyo, Kashiwa 277-8572, Japan.

${ }^{e}$ Department of Physics, Hiroshima University, Higashi-Hiroshima 739-8526, Japan.

${ }^{f}$ Center for Computational Sciences, University of Tsukuba, Tsukuba 305-8577, Japan;

We present a calculation of pion electromagnetic and scalar form factors in two-flavor QCD with the non-perturbatively $O(a)$-improved Wilson fermion. Chiral extrapolation of the corresponding charge radius is discussed based on the chiral perturbation theory.

XXIIIrd International Symposium on Lattice Field Theory

25-30 July 2005

Trinity College, Dublin, Ireland

\footnotetext{
*Speaker.

${ }^{\dagger}$ E-mail: shoji.hashimoto@kek.jp
} 


\section{Introduction}

Electromagnetic and scalar form factors of pion are fundamental quantities in the low energy dynamics of pions. In the chiral perturbation theory (ChPT) their radii are related to the low energy constants at $O\left(p^{4}\right)$. The electromagnetic charge radius is experimentally measured rather precisely, $\left\langle r^{2}\right\rangle_{V}^{\pi}=0.452(10) \mathrm{fm}^{2}[1]$, and the scalar radius can be related to $F_{K} / F_{\pi}$ or $\pi \pi$ scattering amplitudes. Therefore, these simple quantities provide a good testing ground of the lattice calculation techniques. In particular, the chiral extrapolation seems to induce large systematic errors unless the unquenched lattice simulation can treat pions well below $300 \mathrm{MeV}$ where the chiral logarithm effect should become prominent $[2,3]$. It is interesting to check if the lattice data reproduce the expected chiral logarithms in the pion form factors.

Our numerical calculation is performed on the two-flavor gauge ensembles produced by the JLQCD collaboration [4] at $\beta=5.2$ on a $20^{3} \times 48$ lattice with the $O(a)$-improved Wilson fermions. High statistics is the key for the form factor measurements; we accumulated 1,200 gauge configurations for each five sea quark masses. The pion form factors are obtained as a by-product of our calculation of the $K_{l 3}$ form factors [5]. It turned out that the largest effect on the $K_{l 3}$ form factor at zero momentum transfer $f_{+}^{K \pi}(0)$ comes from the shift of the zero recoil point to $t_{\max }=\left(m_{K}-m_{\pi}\right)^{2}$. For the evaluation of $f_{+}^{K \pi}(0) / f_{+}^{K \pi}\left(t_{\max }\right)$ we need a precise knowledge of the pion/kaon charge radius, which is a subject of this paper.

\section{Pion form factors}

The pion electromagnetic form factor $G_{\pi}\left(q^{2}\right)$ is defined as

$$
\left\langle\pi\left(p^{\prime}\right)\left|J_{e m}^{\mu}\right| \pi(p)\right\rangle=G_{\pi}\left(q^{2}\right)\left(p+p^{\prime}\right)^{\mu},
$$

where $q^{2}=\left(p-p^{\prime}\right)^{2}$ and $J_{e m}^{\mu}$ is the electromagnetic current. Because of the charge conservation, the form factor $G_{\pi}\left(q^{2}\right)$ is normalized as $G_{\pi}(0)=1$. It is well known that the experimental data in the small momentum transfer $Q^{2}=-q^{2} \lesssim 1 \mathrm{GeV}^{2}$ are well described by the vector meson dominance hypothesis $G_{\pi}\left(Q^{2}\right)=1 /\left(1+Q^{2} / m_{\rho}^{2}\right)$. (For a summary of experimental results, see for example [6].)

The charge radius $\left\langle r^{2}\right\rangle_{V}^{\pi}$ is a slope of $G_{\pi}\left(q^{2}\right)$ near the zero momentum transfer

$$
G_{\pi}\left(q^{2}\right)=1+\frac{1}{6}\left\langle r^{2}\right\rangle_{V}^{\pi} q^{2}+\cdots
$$

Near the chiral limit, ChPT predicts the chiral logarithm [7]

$$
\left\langle r^{2}\right\rangle_{V}^{\pi}=\frac{12 L_{9}^{r}}{f^{2}}-\frac{1}{(4 \pi f)^{2}}\left[\ln \frac{m_{\pi}^{2}}{\Lambda^{2}}+\frac{3}{2}\right]
$$

Unlike the pion decay constant, for which the chiral logarithm appears in the form $m_{\pi}^{2} \ln m_{\pi}^{2}$, the chiral limit of the charge radius is divergent, and one expects a large effect as the physical pion mass is approached. The current experimental value is $\left\langle r^{2}\right\rangle_{V}^{\pi}=0.452(10) \mathrm{fm}^{2}$ [1].

The scalar form factor $G_{S}\left(q^{2}\right)$ is defined in a similar manner.

$$
\left\langle\left.\pi\left(p^{\prime}\right)\right|^{-} q q \mid \pi(p)\right\rangle=G_{S}\left(q^{2}\right),
$$



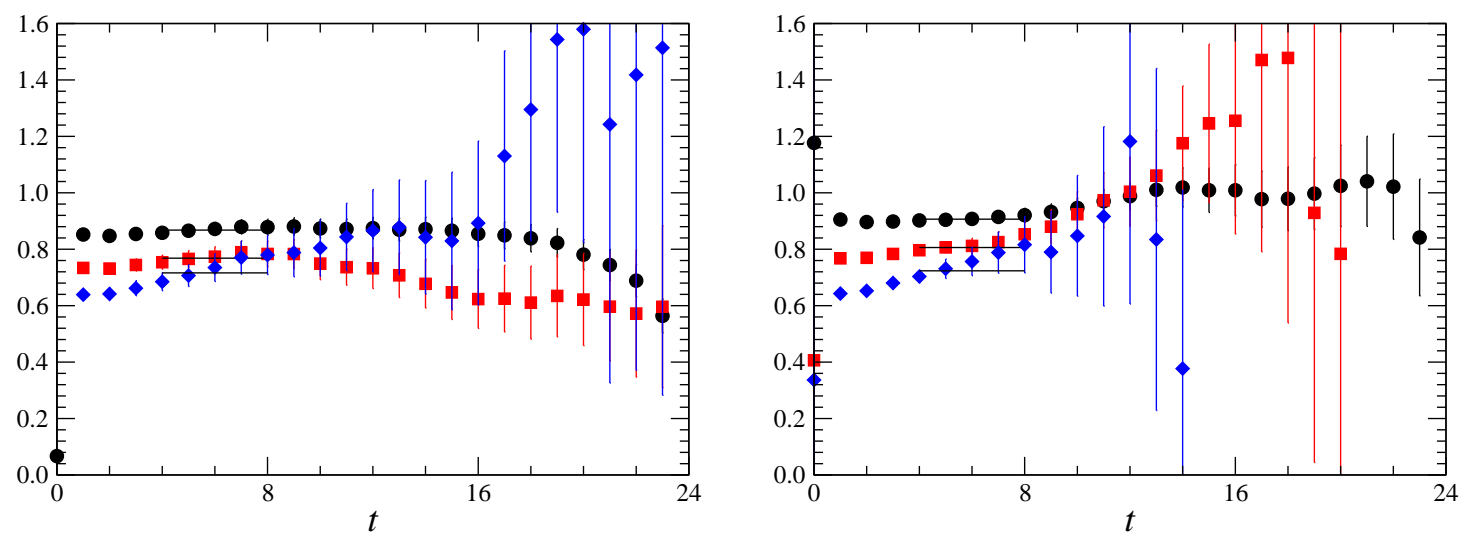

Figure 1: Double ratio (3.1) for the vector form factor at $K=0.1340$ (left) and 0.1355 (right). Momentum $p_{\pi}$ is $(1,0,0)$ (circles), $(1,1,0)$ (squares), and $(1,1,1)$ (diamonds) in units of $2 \pi / L$.

Here the scalar operator is understood as a flavor non-singlet one. The scalar radius $\left\langle r^{2}\right\rangle_{S}^{\pi}$ is defined for $G_{S}\left(q^{2}\right)$ like $\left\langle r^{2}\right\rangle_{V}^{\pi}$ for $G_{\pi}\left(q^{2}\right)$ in (2.3). The chiral logarithm is even stronger for the scalar radius than for the charge radius, i.e. the coefficient of the log term is $-15 / 2(4 \pi f)^{2}$ rather than $-1 /(4 \pi f)^{2}$ in $(2.3)$.

Although there is no direct physical process induced by (2.4), the scalar radius can be extracted from the $\pi \pi$ scattering using ChPT, $\left\langle r^{2}\right\rangle_{S}^{\pi}=0.61(4) \mathrm{fm}^{2}$ [8]. It can also be related to $f_{K} / f_{\pi}$, from which one obtains $0.65(4) \mathrm{fm}^{2}$. For non-degenerate quark masses, the scalar form factor corresponds to $f_{0}^{K \pi}\left(q^{2}\right)$ of the $K_{l 3}$ decay. Its radius $\left\langle r^{2}\right\rangle_{S}^{K \pi}$ is measured as $0.235(14)(8) \mathrm{fm}^{2}$ [9] or $0.165(16) \mathrm{fm}^{2}[10]$ for charged and neutral kaons, respectively. It indicates that the flavor symmetry breaking is a large effect as suggested by the large chiral logarithm.

\section{Lattice calculation}

For the calculation of pion form factors we calculate three-point functions $C^{P^{S} J P^{S}}\left(p_{\pi}, t_{J}\right)$ with operators $J\left(=V_{4}\right.$ or $\left.S\right)$ inserted at time $t_{J}$. Smeared pion interpolating fields $P^{S}$ are set at time $t=0$ and $T / 2$ ( $T$ is the temporal extent of our lattice $T=48$ ). Spatial momentum is inserted at $t=T / 2$ and $t_{J}$, so that the initial pion propagating from $t=0$ to $t_{J}$ is at rest and the final pion between $t_{J}$ and $T / 2$ has a momentum $p_{\pi}$. To extract the form factor we use a double ratio

$$
\frac{\frac{C^{P^{S} J P^{S}}\left(p_{\pi}, t_{J}\right)}{C^{P^{S} J P^{S}}\left(0, t_{J}\right)}}{\frac{C^{P^{S} P^{L}}\left(p_{\pi}, t_{J}\right)}{C^{P^{S} P^{L}}\left(0, t_{J}\right)}} \longrightarrow \frac{G_{J}\left(q^{2}\right)}{G_{J}(0)} \frac{m_{\pi}+E_{\pi}\left(p_{\pi}\right)}{2 m_{\pi}}, \quad 0 \ll t_{J} \ll T / 2,
$$

where the two-point function $C^{P^{S} P^{L}}\left(p_{\pi}, t\right)$ in the denominator is constructed with the same smeared operator $P^{S}$ and a local operator $P^{L}$. In the large enough time separation the numerator gives the ratio of form factors $G_{J}\left(q^{2}\right) / G_{J}(0)$ up to some kinematical factor, while the denominator becomes identity because $\left\langle\pi(p)\left|P^{L}\right| 0\right\rangle /\left\langle\pi(0)\left|P^{L}\right| 0\right\rangle=1$ must be satisfied for Lorentz invariance. Positive and negative $t_{J}$ s are averaged in order to increase statistics. 


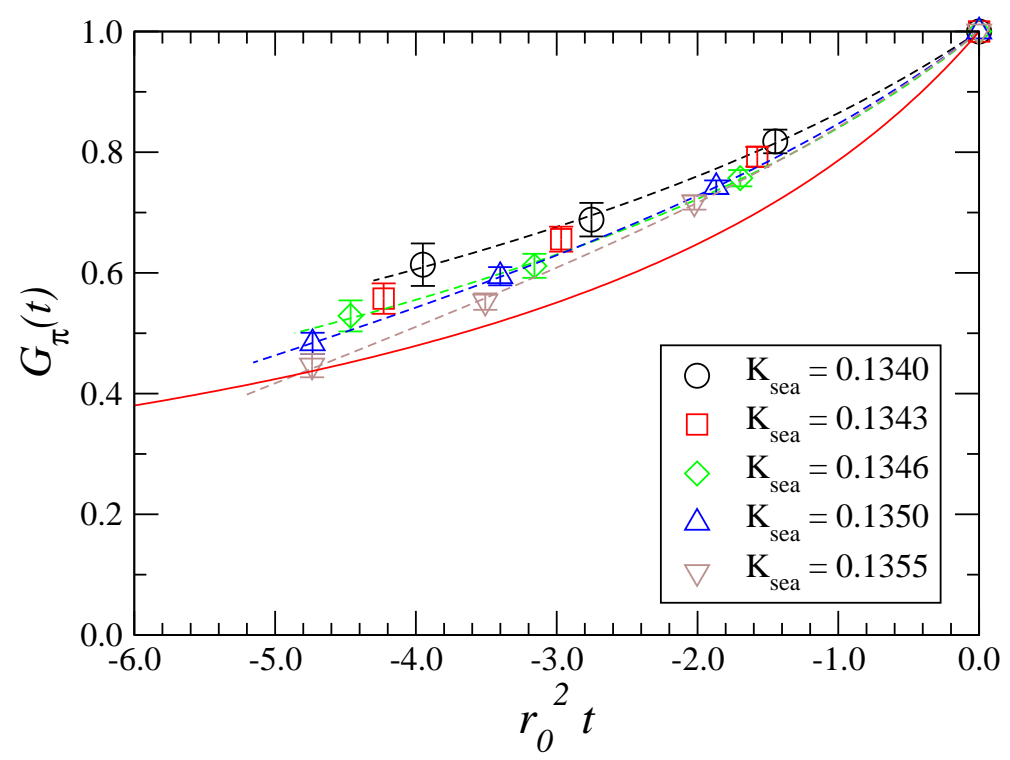

Figure 2: Vector form factor as a function of $t=q^{2}$ normalized by the Sommer scale $r_{0}$. Solid curve shows the vector meson dominance form $1 /\left(1-q^{2} / m_{\rho}^{2}\right)$ with the physical $\rho$ meson mass.

Plots are shown in Figure 1 for the vector current $J=V_{4}$. Results for the heaviest ( $K=0.1340$ ) and lightest $(K=0.1355)$ quark masses are plotted; the data have similar quality for other quark masses. (Sea and valence quark masses are set equal in our calculation setup.) We find a fairly good plateau beyond $t \simeq 4$, though the signal for the largest momentum $(1,1,1)$ is not satisfactory. We fit the data with a constant in the range $[4,8]$ to extract the form factor ratio in the RHS of (3.1).

\section{Form factor results}

In Figure 2 we plot the vector form factor for each $K$ value. We fit the data with two different fit forms:

$$
\begin{aligned}
& G_{\pi}(t)=\frac{1}{1-c_{0}\left(r_{0}^{2} t\right)}+c_{1}\left(r_{0}^{2} t\right)^{2}, \quad \text { (free pole) } \\
& G_{\pi}(t)=\frac{1}{1-t / m_{V}^{2}}+d_{0}\left(r_{0}^{2} t\right), \quad \text { (measured pole) }
\end{aligned}
$$

Here, $t=q^{2}$ is normalized by the Sommer scale $r_{0} \cdot c_{0}, c_{1}$, and $d_{0}$ are fit parameters. Both forms are motivated by the vector meson dominance ansatz, but the pole mass is a free parameter in the "free pole" form (4.1), while the vector meson mass measured on the lattice at a given quark mass is used in the "measured pole" form (4.2). In Figure 2 the fit curves with the "free pole" form are plotted, but both can fit the lattice data equally well.

Theoretically, we prefer the "measured pole" form, because the vector meson pole must appear with the known vector meson mass from the analyticity. There are other contributions from higher resonances and continuum states, that we may parametrize by the linear term $d_{0}\left(r_{0}^{2} t\right)$ near $t=0$. This statement applies when the valence quark mass is heavy enough that the $\rho \rightarrow \pi \pi$ threshold does not open, which is the case in our lattice calculation. As the quark mass decreases, the $\pi \pi$ 


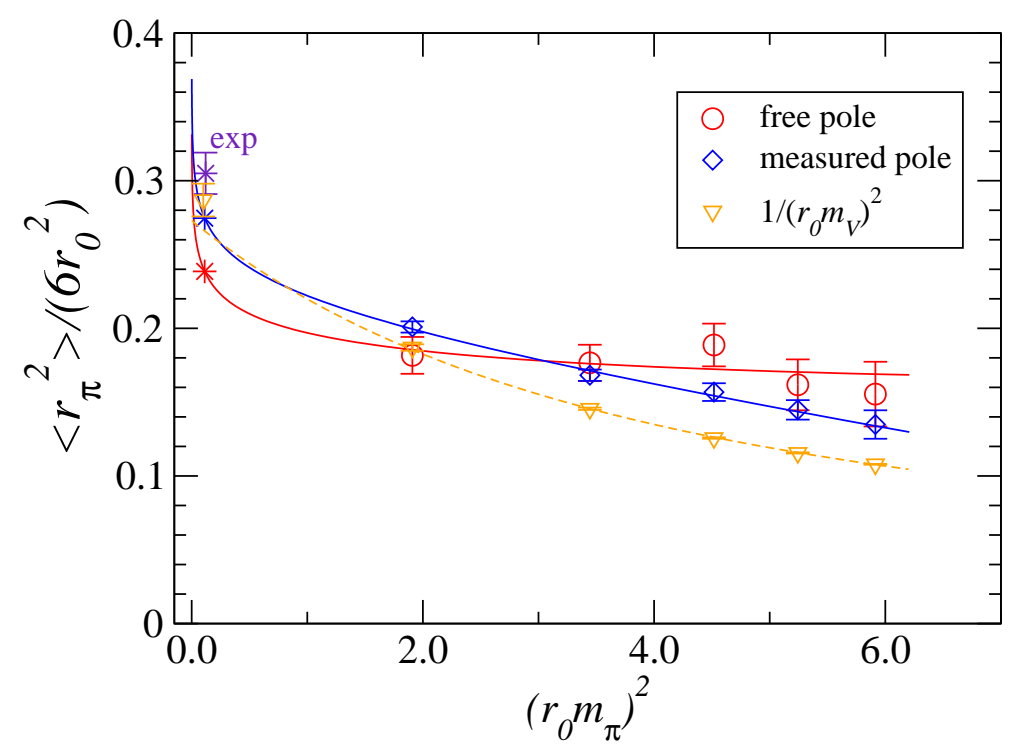

Figure 3: Chiral extrapolation of the pion charge radius. Results from the "free pole" (circles) and "measured pole" (diamonds) are plotted together with the extrapolation curves with (4.3). An expectation from the pole dominance model $1 / m_{V}^{2}$ is also plotted (triangles).

continuum state starts to contribute and finally it reproduces the ChPT prediction near the chiral limit. Therefore, in the physical quark mass regime the pole dominance form should be modified appropriately (see, for example, [11]).

Chiral extrapolation of the pion charge radius is plotted in Figure 3. The "free pole" fit results have essentially no dependence on $m_{\pi}^{2}$ within the large statistical errors. With the "measured pole" fit the statistical error is much reduced and we find an upward trend toward the chiral limit. It is strongly correlated with the pure pole dominance model, which gives $6 / m_{V}^{2}$ and the upward trend is obvious.

For the chiral extrapolation we employ the one-loop ChPT formula with a higher order analytic term

$$
\left\langle r^{2}\right\rangle_{V}^{\pi}=C_{0}-\frac{1}{(4 \pi f)^{2}} \ln \frac{m_{\pi}^{2}}{\mu^{2}}+C_{1} m_{\pi}^{2},
$$

where $C_{0}$ and $C_{1}$ are fit parameters and $\mu$ is an arbitrary scale. We can see that the chiral logarithm enhances the charge radius near the chiral limit, and the value extrapolated to the physical point is $\left\langle r^{2}\right\rangle_{V}^{\pi}=0.396(10) \mathrm{fm}^{2}$, which is about $12 \%$ below the experimental value.

For the scalar form factor we do not expect the dominance of the lowest-lying resonance (the $a_{0}$ meson), as it is already rather far from the $q^{2}=0$ point. We therefore simply fit the form factor by a polynomial. Chiral extrapolation of the scalar radius is shown in Figure 4, where we draw the fit curve with the chiral log form plus a $O\left(m_{\pi}^{2}\right)$ analytic term. The chiral logarithm is very strong for this quantity and the extrapolation could be sensitive to the details of the fit function. Our preliminary result neglecting such systematic effect is $0.60(15) \mathrm{fm}^{2}$.

This work is supported by the Large Scale Simulation Program No. 132 (FY2005) of High Energy Accelerator Research Organization (KEK), and also in part by the Grant-in-Aid of the 


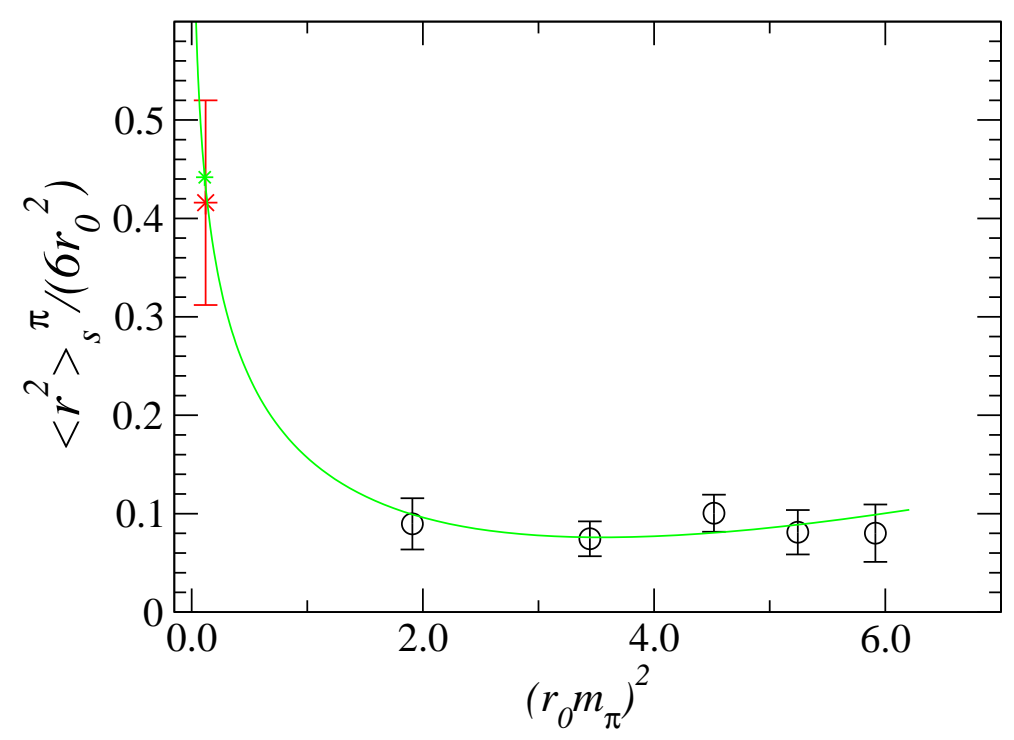

Figure 4: Chiral extrapolation of the scalar radius.

Ministry of Education (Nos. 12740133, 13135204, 13640260, 14046202, 14740173, 15204015, 15540251, 16028201, 16540228, 16740147, 17340066, 17540259).

\section{References}

[1] S. Eidelman et al. [Particle Data Group], Review of particle physics, Phys. Lett. B 592 (2004) 1.

[2] S. Hashimoto et al. [JLQCD Collaboration], Chiral extrapolation of light-light and heavy-light decay constants in unquenched QCD, Nucl. Phys. Proc. Suppl. 119 (2003) 332 [arXiv:hep-lat/0209091].

[3] C. Bernard, S. Hashimoto, D. B. Leinweber, P. Lepage, E. Pallante, S. R. Sharpe and H. Wittig, Panel discussion on chiral extrapolation of physical observables, Nucl. Phys. Proc. Suppl. 119 (2003) 170 [arXiv:hep-lat/0209086].

[4] S. Aoki et al. [JLQCD Collaboration], Light hadron spectroscopy with two flavors of $O(a)$-improved dynamical quarks, Phys. Rev. D 68 (2003) 054502 [arXiv:hep-lat/0212039].

[5] N. Tsutsui et al. [JLQCD Collaboration], in these proceedings.

[6] H. P. Blok, G. M. Huber and D. J. Mack, The pion form factor, arXiv:nucl-ex/0208011.

[7] J. Gasser and H. Leutwyler, Low-energy expansion of meson form-factors, Nucl. Phys. B 250 (1985) 517.

[8] G. Colangelo, J. Gasser and H. Leutwyler, $\pi \pi$ scattering, Nucl. Phys. B 603 (2001) 125 [arXiv:hep-ph/0103088].

[9] O. P. Yushchenko et al., High statistic study of the $K \rightarrow \pi^{0} \mu^{-} v$ decay, Phys. Lett. B 581 (2004) 31 [arXiv:hep-ex/0312004].

[10] T. Alexopoulos et al. [KTeV Collaboration], Measurements of semileptonic $K_{L}$ decay form factors, Phys. Rev. D 70 (2004) 092007 [arXiv:hep-ex/0406003].

[11] F. Guerrero and A. Pich, Effective field theory description of the pion form factor, Phys. Lett. B 412 (1997) 382 [arXiv:hep-ph/9707347]. 